\title{
Defective recruitment, survival and proliferation of bone marrow-derived progenitor cells at sites of delayed diabetic wound healing in mice
}

\author{
M. Albiero - L. Menegazzo • E. Boscaro • C. Agostini • \\ A. Avogaro - G. P. Fadini
}

Received: 9 August 2010 /Accepted: 12 November 2010 /Published online: 17 December 2010

(C) Springer-Verlag 2010

\begin{abstract}
Aims/hypothesis Bone marrow (BM)-derived endothelial progenitor cells (EPC) promote tissue healing and angiogenesis, whereas altered EPC biology may favour diabetic complications. We tested the hypothesis that diabetes impairs the contribution of BM-derived cells at sites of wound healing. Methods Four weeks after induction of diabetes in C57BL/6 mice, hindlimb skin wounds were created and monitored by digital imaging and histology. Circulating EPCs were quantified by flow cytometry before and after wounding. In separate experiments, bone marrow from C57BL/6 mice constitutively producing green fluorescent protein (GFP) was transplanted into myeloablated wild-type mice before induction of diabetes. We quantified proliferation, apoptosis and endothelial differentiation of tissue $\mathrm{GFP}^{+}$cells. Net recruitment of $\mathrm{GFP}^{+}$cells was estimated by correcting the number of tissue $\mathrm{GFP}^{+}$cells at each time point for basal levels, apoptosis and proliferation rates.

Results Diabetes delayed wound healing, with reduced granulation tissue thickness and vascularity, and increased apoptosis. Circulating EPC levels were not modified by 4 week diabetes and/or skin wounding. BM-derived EPCs
\end{abstract}

Electronic supplementary material The online version of this article (doi:10.1007/s00125-010-2007-2) contains supplementary material, which is available to authorised users.

M. Albiero · L. Menegazzo • E. Boscaro • C. Agostini •

A. Avogaro $\cdot$ G. P. Fadini $(\square)$

Department of Clinical and Experimental Medicine,

University of Padova, Medical School,

Via Giustiniani,

2. 35100 Padova, Italy

e-mail: gianpaolo.fadini@unipd.it

M. Albiero • L. Menegazzo • A. Avogaro • G. P. Fadini Venetian Institute of Molecular Medicine,

Padova, Italy
$\left(\mathrm{GFP}^{+} \mathrm{vWf}^{+}\right.$[von Willebrand factor] cells) within the granulation tissue were significantly reduced in diabetic compared with control mice. BM-derived $\mathrm{GFP}^{+}$cells showed increased apoptosis and decreased proliferation in diabetic versus non-diabetic wound tissues. Estimated net recruitment of $\mathrm{BM}$-derived $\mathrm{GFP}^{+}$cells was reduced on day 1 after wounding in diabetic mice.

Conclusions/interpretation Diabetic-delayed wound healing was associated with defective recruitment, survival and proliferation of BM-derived progenitor cells. Local treatments aimed at restoring EPC homing and survival might improve tissue healing in diabetes.

Keywords Chimerism - Green fluorescent protein . Regeneration $\cdot$ Stem cells $\cdot$ Transplantation $\cdot$ Ulcer

$\begin{array}{ll}\text { Abbreviations } \\ \text { BM } & \text { Bone marrow } \\ \text { DM } & \text { Diabetes mellitus } \\ \text { EPC } & \text { Endothelial progenitor cells } \\ \text { FLK } & \text { Fetal liver kinase } \\ \text { GFP } & \text { Green fluorescent protein } \\ \text { HPF } & \text { High power field } \\ \text { KDR } & \text { Kinase insert domain receptor } \\ \text { SDF } & \text { Stromal derived factor } \\ \text { STZ } & \text { Streptozotocin } \\ \text { TRITC } & \text { Tetramethyl rhodamine iso-thiocyanate } \\ \text { vWf } & \text { von Willebrand factor }\end{array}$

\section{Introduction}

Diabetes mellitus predisposes to the development of chronic foot ulcers, which represents a major cause of disability and mortality $[1,2]$. Diabetic neuropathy impairs nociception and 
favours traumatic lesions, whereas diabetic vasculopathy impairs nutritive blood flow and delays wound healing [3]. The mechanisms underlying these alterations include advanced glycation, oxidative stress and widespread microvascular damage [4]. It has been recently shown that, besides damage pathways, vascular regeneration is also important for tissue homeostasis and healing. Interestingly, the bone marrow (BM) harbours a subset of cells capable of endothelial differentiation [5]. These endothelial progenitor cells (EPCs) are released into the peripheral circulation in response to ischaemia or vascular injury; once in the bloodstream, they travel (home) to damaged tissues and participate in endothelial healing and angiogenesis [6]. Diabetic patients with long-standing disease have a severe reduction and dysfunction of circulating EPCs, especially in the presence of peripheral vascular complications and diabetic foot [7]. The impairment of endothelial regenerative cells is indeed considered a novel mechanism of vascular disease in diabetes and might be related to delayed wound healing. Interestingly, transplantation of an EPC-enriched cell population was able to improve ulcer healing more than conventional therapy in a pilot clinical trial in diabetic patients with critical limb ischaemia [8].

We have previously shown that mobilisation of EPCs from the BM after acute ischaemic damage is inhibited by diabetes [9], but it is not clear if the homing process and local survival of these cells are impaired as well. In this study, we used a genetic cell tracking method to assess the contribution of BM-derived cells in a model of delayed diabetic wound healing in terms of recruitment, proliferation and apoptosis.

\section{Methods}

Induction of diabetes and wounding All the procedures involving animals and their care were conducted in accordance with international guidelines, laws and policies and with the National Institutes of Health Principles of Laboratory Animal Care (NIH publication no. 85-23, revised 1985). The protocol was authorised by our local institutions. Ten- to twelve-week-old C57BL6 mice (in-house colony) were used. Diabetes was induced by a single intraperitoneal injection of $150 \mathrm{mg} / \mathrm{kg}$ streptozotocin (STZ) in $\mathrm{pH} 4.5$ citrate buffer. Hyperglycaemia was confirmed after 4 and 7 days by testing blood glucose using a commercially available glucose meter (Glucocard G-Sensor; Menarini, Florence, Italy). Mice with blood glucose $>16.7 \mathrm{mmol} / 1(300 \mathrm{mg} / \mathrm{dl})$ were used. To rule out a persistent effect of STZ on immune/inflammatory blood cells that might bias results from the present study, in separate experiments $(n=6)$ we analysed white blood cell subpopulations, $\mathrm{CD} 34^{+}$cells and apoptotic $\mathrm{CD} 34^{+}$cells at baseline, 3, 5, 7 and 30 days after STZ injection.
After 4 weeks of hyperglycaemia, skin wounds were created on the dorsal surface of the right hindlimb using a $4 \mathrm{~mm}$ punch biopsy device (H-S Medical, Colton, CA, USA). At the time of ulceration, capillary glucose was measured and was confirmed to be $>16.7 \mathrm{mmol} / 1$. Wounds were monitored by taking high resolution frontal photos every 2-3 days using a digital camera (Coolpix; Nikon, Torino, Italy) and the area was calculated relative to a millimetre reference using the image processing software ImageJ (Research Services Branch, National Institute of Mental Health, Bethesda, MD, USA). Diabetic and control animals were used either to determine wound closure times ( $n=8$ per group) or for histological analyses $(n=6$ per group). At baseline, 1 and 4 days after wounding, blood samples were drawn for determination of EPC levels by flow cytometry ( $n=6$ per group). Based on preliminary data, we expected this sample size to be sufficient to detect a $25 \%$ difference in mid-time wound healing, as well as a $30 \%$ difference in granulation tissue thickness. We chose the limb wound in a previously validated type 1 diabetic animals model [10], proven suitable to study the altered diabetic wound healing process.

Bone marrow transplantation In separate experiments, wild-type mice $(n=6)$ were myeloablated with a sublethal dose of intraperitoneal cyclophosphamide $(350 \mathrm{mg} / \mathrm{kg})$. Meanwhile, BM cells were prepared from C57BL/6 mice constitutively producing green fluorescent protein (GFP) (in-house colony) by flushing femurs and tibia. Twentyfour hours after myeloablation, $\mathrm{GFP}^{+}$cells were intravenously infused in myeloablated mice. Four weeks after BM transplantation, recovery and chimerism were assessed by flow cytometry on a peripheral blood sample, by looking at the GFP signal. Then, animals were either injected with STZ $(n=3)$ or vehicle $(n=3)$. Four weeks later, wounds were created as described above and tissues collected on day 1 and 4 and after complete healing. We calculated that this sample size had $80 \%$ power to detect a significant $35 \%$ difference in the number of tissue $\mathrm{GFP}^{+}$cells after wounding in diabetes vs. controls.

Tissue analyses Half-closed time was calculated as the time (in days) after which the wound area was reduced to $50 \%$. Wound tissue and the surrounding 2-4 mm tissues, as well as deep planes, were then removed en bloc for histological analyses and immunofluorescence. The wound tissue block was transversally sectioned at the equator of the wound to standardise the wound area to be analysed. Cryosections, $7 \mu \mathrm{m}$ thick, were stained with haematoxylin and eosin, and Masson's trichrome, using commercially available kits (Bio-Optica, Milan, Italy) according to the manufacturer's instructions. Within each section, wound edges and the granulation tissue were identified under low magnification; 
within the granulation tissue of each section, measurements were performed based on 10 random microscopic fields to minimise operator-dependent selection of areas. Thickness of the granulation tissue was measured in cross-sections, as the connective tissue stained blue with Masson's trichrome between epithelium and the underlying muscle fascia. Vascular density within the granulation tissue was determined by staining with GSL I-isolectin B4 (Vector Labs, Burlingame, CA, USA). Apoptosis of granulation tissue cells was detected with Apoptag Green Plus In situ Apoptosis Detection Kit (Millipore, Vimodrone, Milan, Italy) and counted in ten high-power random fields per slice.

Wound tissues excised from $\mathrm{GFP}^{+} \mathrm{BM}$ transplanted mice were stained with a primary anti-von Willebrand factor (vWf) rabbit polyclonal antibody (dilution 1:150; Abcam, Cambridge, MA, USA) and a secondary TRITC-conjugated anti-rabbit Ig. BM-derived cells in the wound tissue were identified based on the endogenous GFP fluorescence. Total $\mathrm{GFP}^{+}$cells (all BM-derived cells) and $\mathrm{GFP}^{+} \mathrm{vWf}^{+}$cells (BM-derived endothelial cells) were counted in 10 randomly selected high-power fields per slice, and normalised for the degree of chimerism. The percentage of $\mathrm{GFP}^{+}$cells coexpressing vWf were also counted. Apoptosis of $\mathrm{GFP}^{+}$cells was assessed in histological sections using the Apoptag Red Plus In situ Apoptosis Detection Kit (Millipore) and identified as double (red and green) fluorescent nucleated cells. Nuclei were counterstained in blue with Hoechst 33258 (Sigma-Aldrich, Schnelldorf, Germany). Proliferating cells were identified in histological sections by the presence of histone $\mathrm{H} 3$ phosphorylated at serine 10, using a rabbit polyclonal histone $\mathrm{H} 3$ phospho $\mathrm{S} 10$ primary $\mathrm{Ab}$, with dilution 1:250 (ab47297; Abcam, Cambridge, UK) and a secondary swine anti-rabbit TRITC-conjugated polyclonal Ab (Dako Cytomation, Glostrup, Denmark) with dilution 1:100. We counted the number of total proliferating cells (labelled in red) in each section, as well as cells showing double fluorescence for GFP and H3S10, representing proliferating $\mathrm{BM}$-derived cells, in ten random high-power fields per slice.

Quantification of circulating EPCs Mouse circulating EPCs were quantified using flow cytometry on the basis of the expression of the stem cell antigen CD34 and of the endothelial antigen fetal liver kinase (FLK)-1. This phenotype is reminiscent of the $\mathrm{CD} 34^{+} \mathrm{KDR}^{+}$cell population, which is considered the best human EPC phenotype [11]. Peripheral blood was collected before wounding and 4 days later. After erythrocyte lysis, $150 \mu 1$ of blood was incubated with $10 \mu$ l of Alexa Fluor 647 rat $\alpha$-mouse CD34 Ab (Beckton Dickinson, Franklin Lakes, NJ, USA) and $10 \mu \mathrm{l}$ of Alexa Fluor 488 rat $\alpha$-mouse FLK-1 Ab (BioLegend, San Diego, CA, USA). The frequency of peripheral blood cells positive for the above reagents was determined by a twodimensional side scatter fluorescence dot plot analysis, after appropriate gating to exclude granulocytes. Initially we gated CD $34^{+}$peripheral blood cells and then examined the resulting population for dual expression of FLK-1. Mouse white blood cell subpopulations were assessed at different time points after STZ administration by FACS according to side scatter and forward scatter properties of lymphocytes, monocytes and granulocytes. Apoptosis of $\mathrm{CD} 34^{+}$cells was analysed after gating on $\mathrm{CD} 34^{+}$events based on Annexin V (Becton Dickinson) binding to externalised phosphatidylserine. Data were processed using the Macintosh CELLQuest software programme (Beckton Dickinson).

Statistical analysis Data are expressed as mean \pm standard error. Differences between means were assessed using twotailed unpaired Student's $t$ test for two independent groups and paired Student's $t$ test for two repeated measures. When there were more than two repeated measures, we used repeated measures ANOVA with the post hoc Hochberg procedure. Statistical significance was accepted at $p<0.05$.

\section{Results}

Experimental diabetes delays wound healing Healing was monitored macroscopically by quantitative imaging of wound area at different time points. Initial wound area was not different between diabetic and control animals $\left(17.1 \pm 1.0\right.$ vs. $16.8 \pm 1.2 \mathrm{~mm}^{2}$, respectively; $\left.p=0.824\right)$. Figure 1a shows that wound closure was delayed in diabetic mice compared with control mice by about 35 . The residual wound area was significantly higher in diabetic versus control mice on day 4 and $7 \quad(p<0.05$ for both after controlling for multiple testing). On day 7 , wounds were healed in control mice, whereas it took 9-12 days in diabetic mice (Fig. 1b).

Granulation tissue vascularisation is impaired in diabetes The granulation tissue of half-closed wounds was assayed for thickness, vascularity and apoptosis $(n=6$ mice per group). Thickness, measured in Masson's trichrome stained sections, was reduced by about $63 \%$ in diabetic versus control mice $(155 \pm 32$ vs. $412 \pm 48 \mu \mathrm{m}, p=0.02)$. Vascular density, measured by isolectin staining, was reduced by about $60 \%$ in diabetic mice versus control mice ( $77 \pm 18$ vs. $192 \pm 23$ vessels/HPF, $p=0.01$ ). As isolectin may give some non-specific signal within the granulation tissue, counterstaining with vWf was performed, confirming reduced number of Isolectin ${ }^{+} \mathrm{vWf}^{+}$vascular structures in diabetes versus control (see Electronic supplementary material [ESM] Fig. 1). The number of total apoptotic cells 


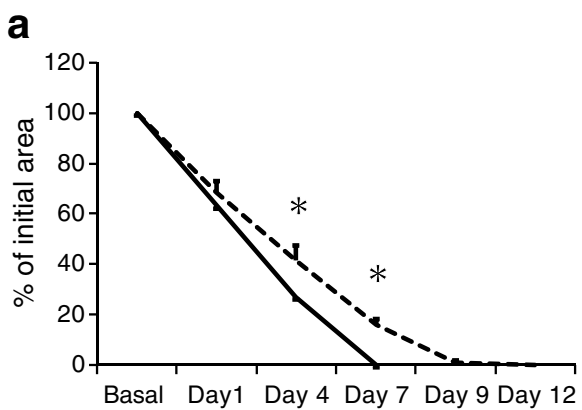

Fig. 1 Experimental diabetes delays wound healing. a Residual wound area was plotted as percentage of initial area at each time point for control (continuous line) and diabetic (dashed line) mice. $p<$

within the granulation tissue was significantly increased in diabetic compared with control mice $(84 \pm 14$ vs. $21 \pm 7$ apoptotic cells/HPF, $p=0.02$ ). Histology showed that most apoptotic cells in control wounds were fibroblasts. Taken together, these data indicated that diabetes impairs development of the granulation tissue with increased apoptosis and decreased vascularisation (Fig. 2).

Skin wounding does not mobilise EPCs Circulating $\mathrm{CD} 34^{+} \mathrm{FLK}-1^{+}$EPCs were quantified by flow cytometry b
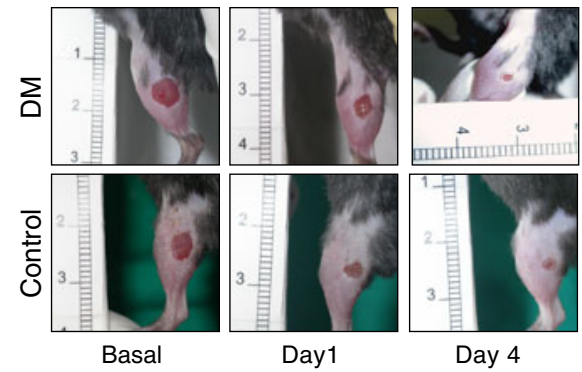

Day 4

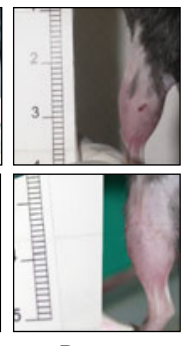

Day 7

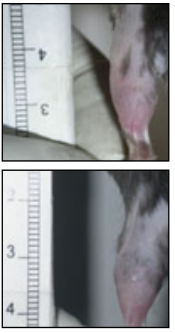

Day 9

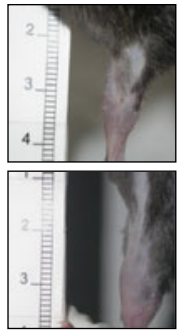

Day 12

0.05 in diabetic versus control mice after controlling for multiple testing. b Representative photographs of wound healing monitoring in diabetic and control mice. DM, diabetes mellitus

before, and on day 1 and 4 after, wounding. We found that 4 weeks of experimental diabetes was associated with a mild and non-significant $20 \%$ reduction in basal EPC levels before wounding. On days 1 and 4 after wounding, EPC level did not change in both control and diabetic mice (ESM Fig. 2a), suggesting that a 4-mm skin wound represents too little tissue damage to stimulate BM EPC mobilisation.

Recruitment, proliferation and apoptosis of BM-derived cells in wound tissues Transplantation of $\mathrm{GFP}^{+} \mathrm{BM}$ into

Fig. 2 Granulation tissue development is impaired in diabetes. a Representative photomicrographs of wound tissue sections stained with: (1) Masson's trichrome for the measurement of thickness (blue area, scale bar $400 \mu \mathrm{m})$; (2) isolectin for identification of blood vessels (green structures); (3) ApopTag Plus for identification of apoptotic cells (green signal; arrowheads identify apoptotic cells). Scale bar, $100 \mu \mathrm{m}$. b-d Quantification of (a) revealed that, compared with control mice (white columns), diabetic mice (black columns) showed significant reduction of granulation tissue thickness and vascularity and significant increase in apoptotic cells. ${ }^{*} p<0.05$ a
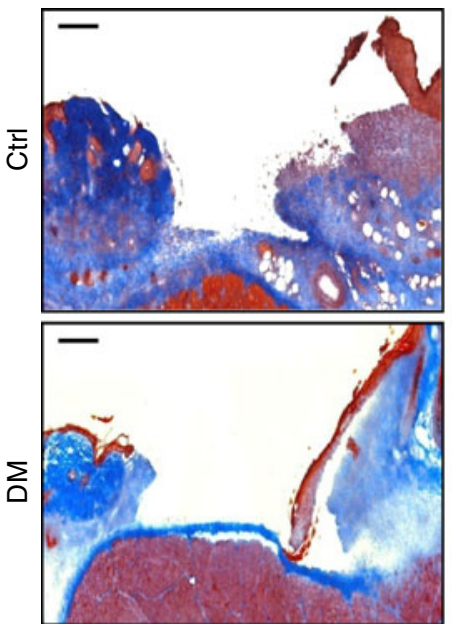

b

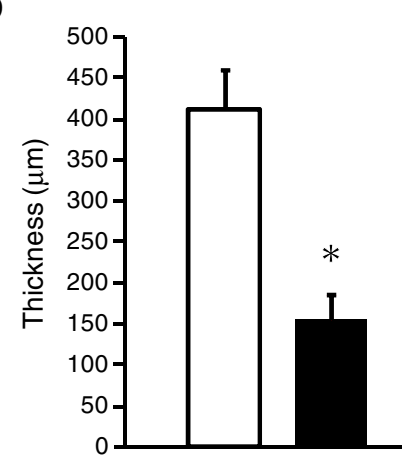

Capillaries
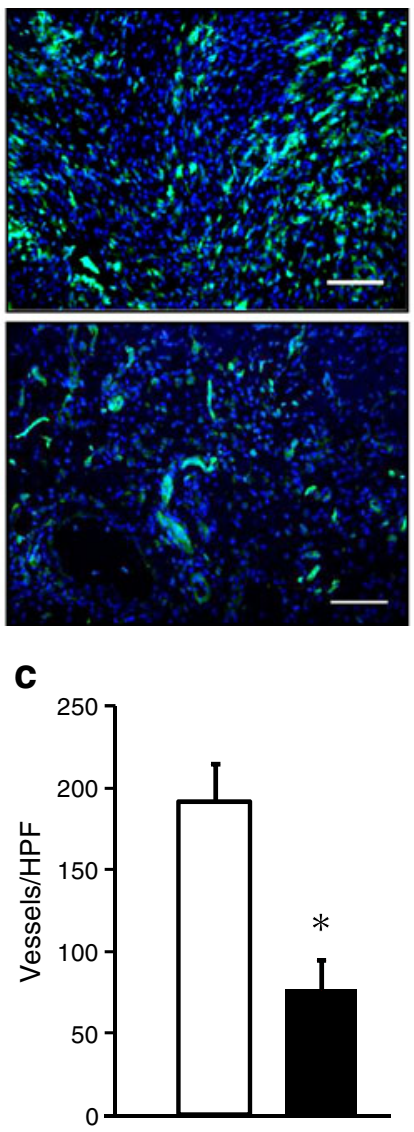

Apoptosis
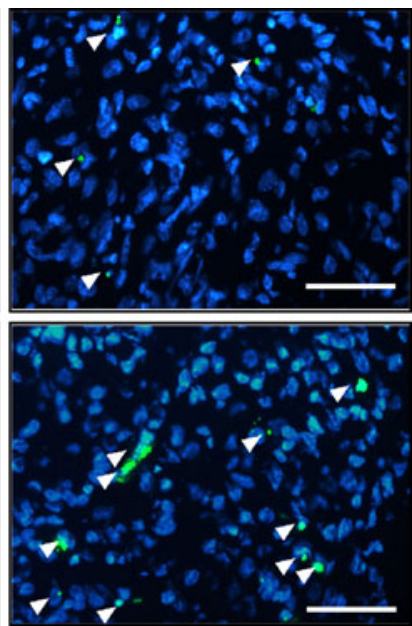

d

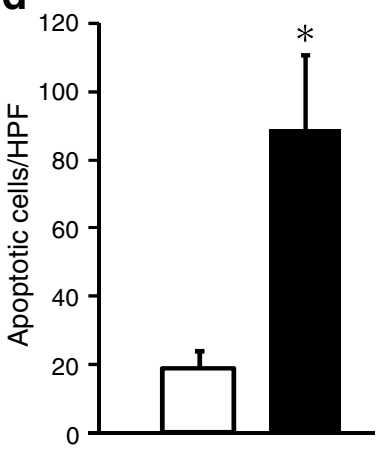


wild-type mice allowed us to track the fate of BM-derived EPCs in diabetic and non-diabetic animals. Administration of STZ to induce diabetes did not significantly modify the percentage of immune/inflammatory blood cells, whereas it induced a transient increase in the percentage of apoptotic CD $34^{+}$cells, which accounted for up to $20 \%$ of total CD $34^{+}$ cells on day 3 after STZ injection. Consistently, the level of $\mathrm{CD} 34^{+}$cells transiently decreased. These alterations resolved after 7 days, and there were no differences on day 30 versus baseline (ESM Fig. 2b). Therefore, acute effects of STZ were confined to the first days after injection, ruling out persistent toxic effects of STZ on the haematopoietic system. In addition, there was no difference in haematopoietic reconstitution between diabetic and control mice.

We found that the number of total $\mathrm{GFP}^{+} \mathrm{BM}$ cells transiently increased after tissue damage, peaking between days 1 and 4 in control mice. A small fraction of these cells in the granulation tissue showed co-expression of $\mathrm{vWf}$, which indicates endothelial differentiation, and therefore identifies BM-derived EPCs. The number of $\mathrm{GFP}^{+}$and $\mathrm{GFP}^{+} \mathrm{vWf}^{+}$ cells were significantly reduced in diabetic mice compared with control mice at all time points after wounding. Interestingly, the percentage of $\mathrm{vWf}^{+} \mathrm{BM}$-derived cells increased to about $15 \%$ in control mice, whereas it remained $<5 \%$ in diabetic mice (Fig. 3).

Theoretically, the difference in $\mathrm{GFP}^{+}$cells during healing of diabetic versus non-diabetic wounds could result from lower proliferation, increased apoptosis or defective recruitment, or a combination of these mechanisms. We found that the apoptotic rate of $\mathrm{GFP}^{+}$cells was slightly but not significantly higher at baseline, while it was much higher in closed wound tissues in diabetic compared with non-

\section{a}
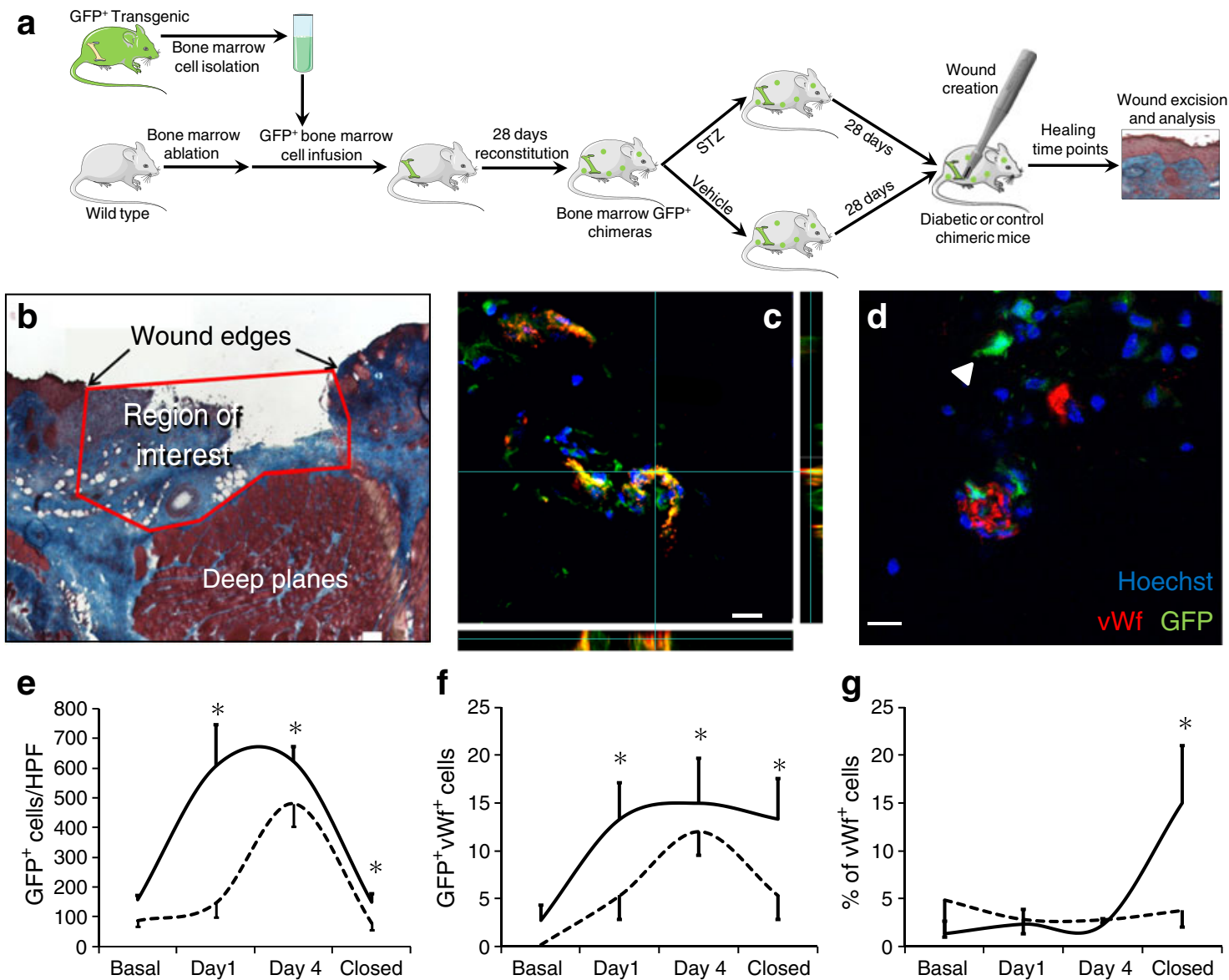

Fig. 3 BM transplantation experiments. a Protocol and time points used to develop $\mathrm{GFP}^{+} \mathrm{BM}$ chimeric mice and identify BM cells homed to wound tissues in diabetic and control mice. b A schematic representation of how the region of interest was defined to locate BMderived cells in a Masson's trichrome stained section. $\mathbf{c}$ Representative confocal immunofluorescence of a tissue section from a non-diabetic wound showing BM-derived cells with endothelial differentiation: GFP signal (green) colocalising with vWf signal (red) appears in yellow. Right-hand and bottom strips showing lateral views of the section (total thickness $7 \mu \mathrm{m}$ ) focused on the horizontal and vertical axis confirm that GFP and vWf signals come from the same cells (scale bar $12 \mu \mathrm{m}$ ). d Representative confocal immunofluorescence of a tissue section from a diabetic wound showing BM-derived cells without endothelial differentiation and no colocalisation of GFP (arrowhead) and vWf signals (scale bar $24 \mu \mathrm{m}$ ). Nuclei counterstained in blue with Hoechst. e-g Time course of total $\mathrm{GFP}^{+}(\mathbf{e}), \mathrm{GFP}^{+} \mathrm{vWf}^{+}$ (f) and $\mathrm{vWf}^{+}(\mathrm{g})$ cells at sites of wound healing in control (continuous line) and diabetic (dashed line) mice. $p<0.05$ in diabetes versus control mice after correction for multiple testing. Scale bar, $50 \mu \mathrm{m}$ 
diabetic animals $(28.5 \pm 11.8$ vs. $0.2 \pm 0.0 p=0.02$; Fig. $4 a)$. The proliferation rate of dermal cells was close to zero at baseline in non-wounded tissues, while it increased substantially after wounding in control animals. Diabetic wound tissues had a lower number of proliferating cells on day 4 $(p=0.03)$ and in closed wounds $(p=0.02)$; proliferating $\mathrm{GFP}^{+}$ cells were significantly reduced in diabetic compared with non-diabetic tissues on day $1(0.3 \pm 0.3$ vs. $13.5 \pm 7.4 p=0.03)$ and 4 ( $1.4 \pm 0.6$ vs. $8.5 \pm 4.5 p=0.02$; Fig. $4 \mathrm{~b})$.

We reasoned that increased apoptosis and reduced proliferation contributes to explaining the lower number of $\mathrm{GFP}^{+}$cells in diabetic wound tissues. To estimate the actual contribution of defective recruitment at each time point, tissue $\mathrm{GFP}^{+}$cell counts were reduced by the percentage of proliferating cells (because proliferation increases the number of local $\mathrm{GFP}^{+}$cells independently of recruitment) and augmented by the percentage of apoptotic cells (because apoptosis reduces the number of local $\mathrm{GFP}^{+}$ cells that may have been recruited). Then, we subtracted the basal $\mathrm{GFP}^{+}$cell count (ESM Fig. 3a-c). The resulting adjusted recruitment curve showed a defective income of $\mathrm{GFP}^{+}$cells in diabetic versus non-diabetic animals on day 1 (ESM Fig. 3d).
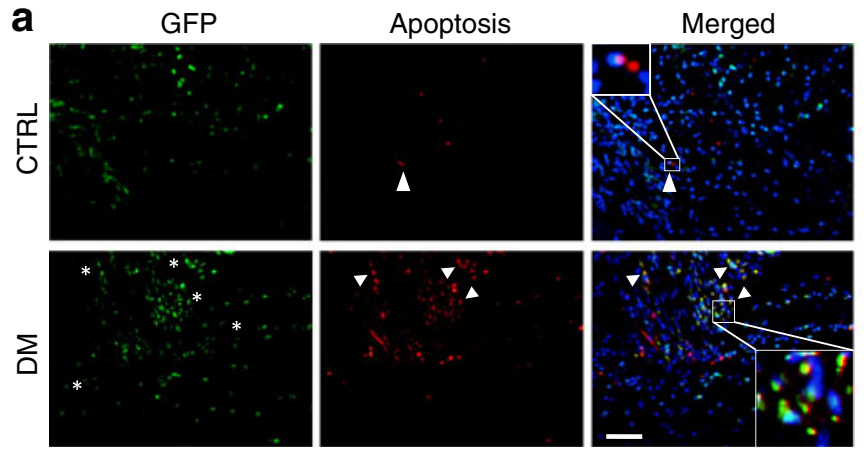

C

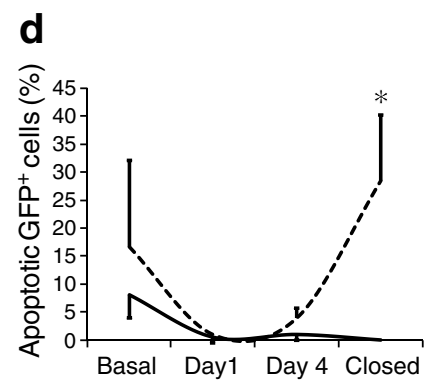

Fig. 4 Apoptosis and proliferation of BM-derived cells. a Apoptosis of $\mathrm{GFP}^{+}$cells was assessed before and at different time points after wounding in diabetic and non-diabetic tissues. Cells showing ApopTag red fluorescence colocalised with GFP fluorescence were considered apoptotic BM-derived cells. In an example from a control wound, apoptotic cells were not derived from the BM as there was no red/green colocalisation (insert). An example from a diabetic wound section shows several $\mathrm{GFP}^{+}$BM-derived cells with red apoptotic signal (insert). Scale bar $50 \mu \mathrm{m}$. b Proliferation of $\mathrm{GFP}^{+}$cells was assessed before and at different time points after wounding in diabetic

\section{Discussion}

In the present study, we used an in vivo genetic cell tracking method to demonstrate that impaired wound healing and vascularisation of the granulation tissue is associated with defects of BM-derived EPCs in diabetic mice, comprising lower proliferation, increased apoptosis and defective recruitment.

During the last 10 years, data have accumulated indicating that reduction and impairment of EPCs are involved in the development of diabetic complications [12]. In humans, circulating EPCs are altered in parallel with increasing severity of micro- and macrovascular disease [7, 13]. In addition, a low EPC level has been shown to represent an independent risk factor for future cardiovascular events $[14,15]$. These clinical data support, but do not prove, the idea that EPCs influence diabetic complications and outcomes. Experimental studies show that restoration of a normal EPC pool is associated with prevention or reversal of myocardial and peripheral ischaemia, as well as glomerular and retinal damage in diabetic animals [16-19], mechanistically demonstrating the protective role of EPCs. Preliminary clinical experience
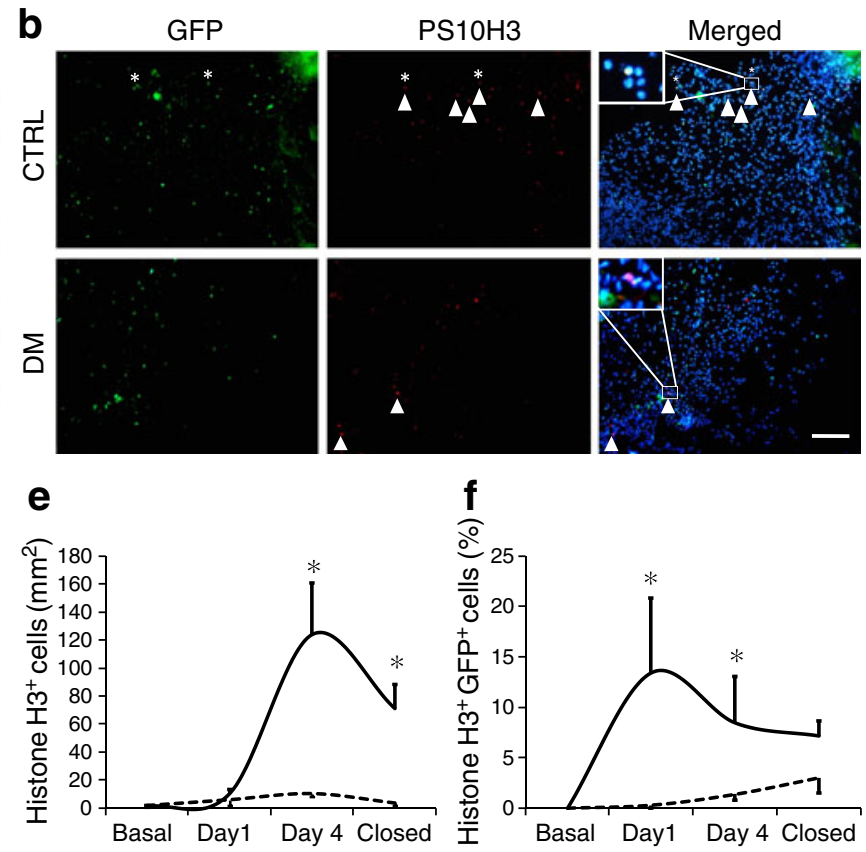

and non-diabetic tissues. Cells showing red phospho-histone $\mathrm{H} 3$ (PS10H3) and green GFP double fluorescence were considered proliferating $\mathrm{GFP}^{+}$cells. c-d Total apoptotic cells and $\mathrm{GFP}^{+}$apoptotic cells are plotted versus time for diabetic (dashed line) and non-diabetic (continuous line) animals. e-f Total proliferating cells and $\mathrm{GFP}^{+}$ proliferating cells are plotted versus time for diabetic (dashed line) and non-diabetic (continuous line) animals. ${ }^{*} p<0.05$ vs. basal after correction for multiple testing. Scale bar, $100 \mu \mathrm{m}$. CTRL, control mouse; DM, diabetic mouse 
confirms that EPC-based therapies are safe and potentially effective in treating diabetic complications, such as critical limb ischaemia [8].

The causes of low EPC levels in diabetes are not entirely understood. We hypothesise a three-compartment model of EPC kinetics, made up of a reservoir (BM), a disposable pool (peripheral blood) and a target (peripheral tissues). Thus, the theoretical mechanisms accounting for circulating EPC reduction include: (1) defective bone marrow mobilisation; (2) death in the bloodstream (e.g. apoptosis); and (3) increased homing outside the bloodstream (Fig. 5). It has been previously shown that post-ischaemic angiogenesis and mobilisation of EPCs are impaired in diabetic rats and mice, owing to an inability to upregulate hypoxiasensing systems in ischaemic tissues and reduced BM responsiveness $[9,20,21]$. More recently, BM histopathologies have been substantiated in diabetic animals, with evidence of microangiopathy and neuropathy [19, 22]. Reduced survival or differentiation of EPCs cultured in high glucose have been shown by several groups in vitro $[23,24]$, but there is no evidence that this occurs in vivo. Our recent study in humans supports the argument against an increased apoptosis of circulating progenitor cells from diabetic patients compared with controls [25]. Finally, low EPCs might reflect an increased homing to target tissues owing to the widespread endothelial damage in diabetes. This 'waste' hypothesis has not been demonstrated so far.

In the present study, we used a mouse model of relatively short disease duration (4 weeks), which was not sufficient to produce a significant reduction of circulating EPCs in mice. Probably, these mice had not yet developed anatomical BM alterations, which have been shown only after 24 weeks of hyperglycaemia [22]. In addition, the degree of tissue damage produced by a $4 \mathrm{~mm}$ skin wound was not sufficient to trigger BM mobilisation of EPCs in diabetic or control mice. As a result, circulating EPC levels were similar in control and diabetic mice throughout the study. This is an ideal situation to study homing processes, indeed, changes in the amount of local EPCs after tissue damage could be attributed to altered recruitment from the bloodstream, proliferation or survival. We found that BMderived EPCs $\left(\mathrm{GFP}^{+} \mathrm{vWf}^{+}\right.$cells) were significantly reduced in diabetic wound tissues compared with non-diabetic ones. To account for this alteration, in mid-late time points of wound healing, apoptosis was higher whereas proliferation of BM-derived $\mathrm{GFP}^{+}$cells was lower in diabetic animals compared with controls. Indeed, the number of local BMderived cells at any time point represents the balance between recruitment and proliferation (that increase it) and apoptosis (that lowers it). The following formula can be applied: number of cells in sections $=$ basal cell count + recruited cells + proliferated cells - apoptotic cells. Therefore, the net recruitment was estimated by subtracting the basal cell count and proliferation rate and by adding the apoptotic rate to the number of local $\mathrm{GFP}^{+}$cells. With this approach, we found a defective recruitment at the earliest time point (day 1) in diabetic wound tissues compared with non-diabetic wound tissues. Proliferation and apoptosis

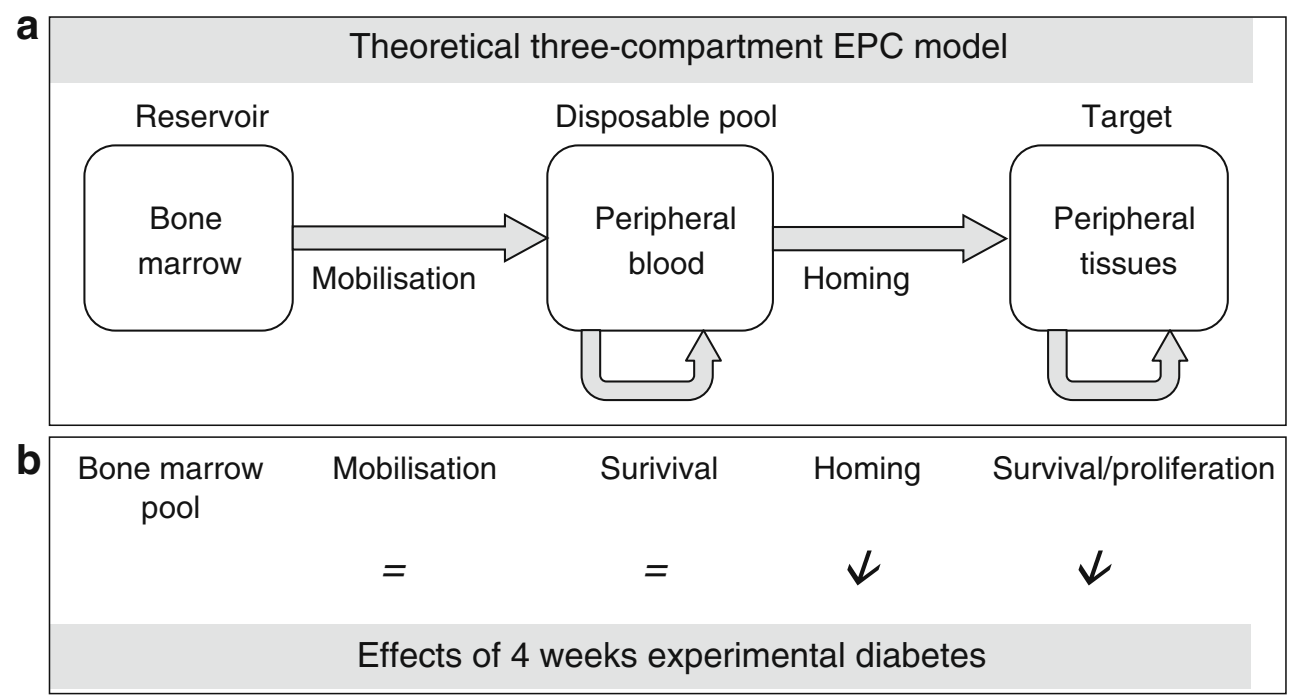

Fig. 5 The three-compartment model of EPC kinetics. a According to current knowledge, EPCs move from a reservoir (BM) to a disposable pool (peripheral blood) through processes of mobilisation. Survival of EPCs in the bloodstream can then be differentially regulated. From the blood, EPCs can reach the target compartment (peripheral tissues) by homing/recruitment processes. Circulating EPC levels are positively influenced by BM mobilisation and peripheral survival of EPCs, whereas it is negatively affected by increased homing. Within the target tissue, the level of EPCs results from the balance between recruitment, survival and proliferation. b The effects of 4 week experimental diabetes on the three compartment kinetics 
showed plausible and consistent variations over time. For instance, in normal unwounded skin, dermal cell proliferation was virtually absent and there was a mildly increased apoptotic rate in diabetes. Proliferation increased after wounding as part of the granulation tissue development. Apoptosis of $\mathrm{GFP}^{+}$cells decreased due to the effect of the incoming (non-apoptotic) BM-derived cells recruited from the bloodstream after wounding.

We identified BM-derived EPCs as $\mathrm{GFP}^{+}$cells coexpressing $\mathrm{vWf}$, as an index of endothelial differentiation. In this study, we used different definitions for circulating EPCs $\left(\mathrm{CD} 34^{+} \mathrm{FLK}-1^{+}\right)$and local EPCs $\left(\mathrm{GFP}^{+} \mathrm{vWf}^{+}\right)$because CD34 is rapidly downregulated in tissue-resident cells and because FLK-1 is expressed in more immature cells than vWf [26]. Thus, local $\mathrm{GFP}^{+} \mathrm{vWf}^{+}$cells may represent BMderived EPCs that home to the tissue and complete endothelial maturation or BM-derived cells that differentiate toward the endothelial lineage within the tissue. In both cases, these cells are known to contribute to granulation tissue angiogenesis [27]. In control and diabetic mice, the total number of $\mathrm{GFP}^{+}$cells peaked during wound healing and then returned to baseline in completely healed tissues. By contrast, $\mathrm{GFP}^{+} \mathrm{vWf}^{+}$cells increased steadily in control mice, while they were lower, and only transiently increased, in diabetic mice (Fig. 3f). Remarkably, the percentage expression of $\mathrm{vWf}$ on $\mathrm{GFP}^{+}$cells, reflecting the accumulation of BM-derived EPCs or progressive endothelial differentiation of BM-derived cells within the granulation tissue, was strikingly flattened in diabetic wounds (Fig. 3g). The reduction of EPCs in diabetic tissues was associated with a severely compromised development of granulation tissue. Given the low absolute number of $\mathrm{GFP}^{+} \mathrm{vWf}^{+}$cells, it is possible that these cells contribute to the vascular bed mainly by paracrine activity (i.e., by secretion of growth factors and cytokines) rather than physically forming entire capillaries [28]. Nonetheless, neovasculogenesis is essential for the formation of a competent connective tissue for healing of a substance loss [3], because impairment of nutritive blood flow hampers cell proliferation and differentiation, while promoting apoptosis. The result of these histopathological alterations was delayed wound healing in diabetic mice. Although we have not definitely demonstrated that EPC defects account for poor granulation tissue vascularisation in diabetes, data in the literature support this view, and restoration of EPC homing and survival in diabetic tissues may improve local neovasculogenesis and tissue healing. For instance, it was previously shown that human BM-derived stem cells accelerate vascularisation and healing of murine diabetic wounds [29]. In addition, Barcelos et al. [29] found that local treatment with exogenous human vascular stem cells improves healing of mouse diabetic wounds by paracrine stimulation of angiogenesis. Our data show defects of endogenous BM-derived stem cells in diabetic wound healing provide a hitherto unrecognised rationale for devising similar therapeutic strategies.

The mechanism underlying the defective contribution of BM-derived cells in diabetes needs to be further explored. Previous data indicate that production of the chemokine SDF (stromal derived factor)- $1 \alpha$, an established regulator of EPC trafficking, is blunted in diabetic tissues [9]. Gallagher et al. [20] reported that local treatment with SDF-1 $\alpha$ improved wound healing and increased local BM-derived cells. While chemokine defects/imbalances may account for altered EPC recruitment, multiple factors are likely to be implicated in the complex impairment of BM-derived cells in diabetic wound healing; these may include biochemical pathways activated by hyperglycaemia together with oxidative stress, accounting for increased local apoptosis. In parallel p53/p21 signalling in high glucose may trigger early EPC senescence and proliferative arrest [30].

In conclusion, we show that diabetes impairs the contribution of BM-derived cells to the wound healing process, by means of increased apoptosis, reduced proliferation and defective recruitment.

The demonstration of impaired homing of EPCs to sites of delayed wound healing identifies another mechanism that hampers wound healing in diabetes. Thus, for the first time, we provide a rationale for devising therapeutic strategies aimed at enhancing progenitor cell recruitment and survival to achieve good vascularisation of the granulation tissue. This might finally translate into improved outcomes of this disabling diabetic complication.

Acknowledgements G.P. Fadini is supported by a European Foundation for the Study of Diabetes (EFSD) young investigator grant and by a EFSD/Lilly Fellowship.

Duality of interest The authors declare that there is no duality of interest associated with this manuscript.

\section{References}

1. Ramsey SD, Newton K, Blough D et al (1999) Incidence, outcomes, and cost of foot ulcers in patients with diabetes. Diabetes Care 22:382-387

2. Faglia E, Clerici G, Clerissi J et al (2009) Long-term prognosis of diabetic patients with critical limb ischemia: a population-based cohort study. Diabetes Care 32:822-827

3. Yamaguchi Y, Yoshikawa K (2001) Cutaneous wound healing: an update. J Dermatol 28:521-534

4. Avogaro A, de Kreutzenberg SV, Fadini G (2008) Endothelial dysfunction: causes and consequences in patients with diabetes mellitus. Diabetes Res Clin Pract 82(Suppl 2):S94-S101

5. Asahara T, Murohara T, Sullivan A et al (1997) Isolation of putative progenitor endothelial cells for angiogenesis. Science 275:964-967

6. Takahashi T, Kalka C, Masuda H et al (1999) Ischemia- and cytokine-induced mobilization of bone marrow-derived endothelial progenitor cells for neovascularization. Nat Med 5:434-438 
7. Fadini GP, Sartore S, Albiero M et al (2006) Number and function of endothelial progenitor cells as a marker of severity for diabetic vasculopathy. Arterioscler Thromb Vasc Biol 26:2140-2146

8. Huang P, Li S, Han M et al (2005) Autologous transplantation of granulocyte colony-stimulating factor-mobilized peripheral blood mononuclear cells improves critical limb ischemia in diabetes. Diabetes Care 28:2155-2160

9. Fadini GP, Sartore S, Schiavon M et al (2006) Diabetes impairs progenitor cell mobilisation after hindlimb ischaemia-reperfusion injury in rats. Diabetologia 49:3075-3084

10. Fadini GP, Albiero M, Menegazzo L et al (2010) The redox enzyme p66Shc contributes to diabetes and ischemia-induced delay in cutaneous wound healing. Diabetes 59:2306-2314

11. Fadini GP, Baesso I, Albiero M et al (2008) Technical notes on endothelial progenitor cells: ways to escape from the knowledge plateau. Atherosclerosis 197:496-503

12. Fadini GP, Sartore S, Agostini C, Avogaro A (2007) Significance of endothelial progenitor cells in subjects with diabetes. Diabetes Care 30:1305-1313

13. Dessapt C, Karalliedde J, Hernandez-Fuentes M et al (2010) Circulating vascular progenitor cells in patients with type 1 diabetes and microalbuminuria. Diabetes Care 33:875-877

14. Werner N, Kosiol S, Schiegl T et al (2005) Circulating endothelial progenitor cells and cardiovascular outcomes. N Engl J Med 353:999-1007

15. Fadini GP, de Kreutzenberg S, Agostini C et al (2009) Low CD34 + cell count and metabolic syndrome synergistically increase the risk of adverse outcomes. Atherosclerosis 207:213-219

16. Tuo QH, Zeng H, Stinnett A et al (2008) Critical role of angiopoietins/Tie-2 in hyperglycemic exacerbation of myocardial infarction and impaired angiogenesis. Am J Physiol Heart Circ Physiol 294:H2547-H2557

17. Tamarat R, Silvestre JS, Le Ricousse-Roussanne S et al (2004) Impairment in ischemia-induced neovascularization in diabetes: bone marrow mononuclear cell dysfunction and therapeutic potential of placenta growth factor treatment. Am J Pathol $164: 457-466$

18. Remuzzi G, Benigni A, Remuzzi A (2006) Mechanisms of progression and regression of renal lesions of chronic nephropathies and diabetes. J Clin Invest 116:288-296
19. Busik JV, Tikhonenko M, Bhatwadekar A et al (2009) Diabetic retinopathy is associated with bone marrow neuropathy and a depressed peripheral clock. J Exp Med 206:2897-2906

20. Gallagher KA, Liu ZJ, Xiao M et al (2007) Diabetic impairments in NO-mediated endothelial progenitor cell mobilization and homing are reversed by hyperoxia and SDF-1 alpha. J Clin Invest 117:1249-1259

21. Ceradini DJ, Yao D, Grogan RH et al (2008) Decreasing intracellular superoxide corrects defective ischemia-induced new vessel formation in diabetic mice. J Biol Chem 283:10930-10938

22. Oikawa A, Siragusa M, Quaini F et al (2010) Diabetes mellitus induces bone marrow microangiopathy. Arterioscler Thromb Vasc Biol 30:498-508

23. Marchetti V, Menghini R, Rizza S et al (2006) Benfotiamine counteracts glucose toxicity effects on endothelial progenitor cell differentiation via Akt/FoxO signaling. Diabetes 55:2231-2237

24. Seeger FH, Haendeler J, Walter DH et al (2005) p38 mitogenactivated protein kinase downregulates endothelial progenitor cells. Circulation 111:1184-1191

25. Fadini GP, Boscaro E, de Kreutzenberg S et al (2010) Time course and mechanisms of circulating progenitor cell reduction in the natural history of type 2 diabetes. Diabetes Care 33:1097-1102

26. Hristov M, Erl W, Weber PC (2003) Endothelial progenitor cells: isolation and characterization. Trends Cardiovasc Med 13:201206

27. Tepper OM, Capla JM, Galiano RD et al (2005) Adult vasculogenesis occurs through in situ recruitment, proliferation, and tubulization of circulating bone marrow-derived cells. Blood 105:1068-1077

28. Barcelos LS, Duplaa C, Krankel N et al (2009) Human CD133+ progenitor cells promote the healing of diabetic ischemic ulcers by paracrine stimulation of angiogenesis and activation of Wnt signaling. Circ Res 104:1095-1102

29. Sivan-Loukianova E, Awad OA, Stepanovic V, Bickenbach J, Schatteman GC (2003) CD34+ blood cells accelerate vascularization and healing of diabetic mouse skin wounds. J Vasc Res 40:368-377

30. Rosso A, Balsamo A, Gambino R et al (2006) p53 Mediates the accelerated onset of senescence of endothelial progenitor cells in diabetes. J Biol Chem 281:4339-4347 